\title{
Effectiveness of a Mass Immunization Campaign Against Serogroup C Meningococci in Children in the Federal State of Santa Catarina, Brazil
}

\author{
Emil Kupek, Rubens C. B. Puricelli \\ and Maria Helena B. Westrupp
}

Federal University of Santa Catarina, Department of Public Health; Health Secretary of the State of Santa Catarina, SC, Brazil

\begin{abstract}
In addition to vaccine efficacy studies, there is a pressing need to evaluate vaccine effectiveness in a way that takes into account the limitations of health care systems in certain settings. An attempt to reach this objective was exemplified by a vaccination campaign against serogroup $C$ meningococci in the federal state of Santa Catarina, in Brazil. A polysaccharide vaccine against serogroup $C$ meningococci was administered to all individuals between 6 months and 14 years of age in March, 1996, in the municipalities that had the highest incidence of meningococcal disease in the previous year. All cases of the disease due to this serogroup observed in Santa Catarina during a 1-year period before and after the vaccination were included in the analysis. The cumulative incidence rate ratio was calculated for the unvaccinated compared to the vaccinated area. As a second step, the ratio of this quantity for the period before and after the vaccination, i.e. the ratio of the rate ratios (RRR), was calculated. One minus RRR was used to estimate the vaccine effectiveness. In the general population, the vaccine effectiveness was $74.3 \%(95 \%$ confidence intervals $52.7 \%$ to $99.6 \%$ ). In children 6 months to 14 years, vaccine effectiveness was $93.1 \%(85.2 \%$ to $100 \%)$. Vaccine effectiveness could not be confirmed within more specific age bands, probably due to the lack of statistical power. It is concluded that group $\mathrm{C}$ meningococcal vaccine is effective in reducing the occurrence of meningococcal disease in children 6 months to 14 years of age, and that the ratio of rate ratios (RRR) in a useful method to evaluate vaccine effectiveness.
\end{abstract}

Key Words: Vaccine, effectiveness, meningitis, meningococcal disease, serogroup C, Brazil.

This study analysed the effectiveness of a mass immunization campaign against meningococcal disease applied to a selected area in the federal state of Santa Catarina, in southern Brazil. We present relevant epidemiological data, followed by a discussion of methodological issues related to vaccine effectiveness in this context, a statistical analysis, and an example using relevant data.

Received on 28 June 2001; revised 27 December 2001.

Address for correspondence: Prof. Dr. Emil Kupek. Departamento de Saúde Pública, CCS. Universidade Federal de Santa Catarina, Campus Universitário, Trindade. Zip Code: 88040-900 Florianópolis-SC, Brazil. Phone: (55 48) 334-8654. Fax: (55 48) 331-9542. E-mail: kupek@repensul.ufsc.br

The Brazilian Journal of Infectious Diseases 2001 ;5(6):324-331. (C) 2001 by The Brazilian Journal of Infectious Diseases and Contexto Publishing. All rights reserved. 1413-8670
In 1974, Brazil was hit by an epidemic of meningococcal disease caused predominantly by serogroups A and C of Neisseria meningitidis [1] which resulted in an annual incidence of more than 30 per 100,000 inhabitants [2]. A year later, a mass vaccination campaign reached 80 million people [1] and reduced the incidence, but it bounced back to the epidemic level in 1976, before stabilizing at an endemic level in subsequent years [3]. A new epidemic of meningococcal disease in 1988 was caused primarily by serogroup B, and motivated the Brazilian Ministry of Health to vaccinate almost 4 million children against serogroups B and C by the end of the decade [1]. During the 1990 to 1996 period, annual incidence did not pass the 5 per 100,000 level in the general population, although it was in the range of 20 per 100,000 for children under one year of age [3]. 
Although both efficacy and effectiveness of polysaccharide anti-meningococcal vaccines have been confirmed for older children and adolescents in various countries [4-8], including Brazil [9-11], some methodological difficulties seem to have hampered routine evaluation of their beneficial effects. First, the administration of vaccine compliant to a gold standard for vaccine efficacy (double-blind randomized control trials (RCT) with randomized selection of high risk participants, ideal vaccine storage conditions, intensive monitoring, and strict supervision) is very difficult to maintain in health service practice, particularly in developing countries. This led some authors to emphasize effectiveness rather than efficacy studies as criterion for usefulness of a vaccine in a particular setting [12]. Second, selection bias due to exclusion of patients who died before reaching the hospital and were less likely to be vaccinated may have underestimated antimeningococcal vaccine efficacy in two Brazilian casecontrol studies $[9,11]$, as pointed out by Costa $[10,13]$. These factors are difficult to control in health care settings in most developing countries. Third, better use of existing surveillance data and improving data quality can provide important monitoring information on viable prevention strategies, although it cannot serve as a substitute for RCT evaluation studies. This is particularly important when risk factors change in a short period of time; for example, when new strains or interventions such as vaccination campaigns are introduced in specific areas but their impact may spread beyond these areas.

The above difficulties were experienced when looking for a suitable vaccine effectiveness measure for a mass vaccination campaign in 42 municipalities with the highest incidence of meningococcal disease in the federal state of Santa Catarina, in March, 1996. The state maintained an elevated incidence of meningococcal disease compared to the national average throughout the decade of 1990, varying between 6 and 11 cases per 100,000 inhabitants annually with a case-fatality rate in the range of $9 \%$ to 17\% [14]. From 1990 to 1995 , the percentage of serogroup C meningococci increased from $11 \%$ to over half of the cases serogrouped. This prompted the decision by the health authorities to vaccinate all children between 6 months and 14 years of age in 42 municipalities where $84 \%$ of cases and $73 \%$ of fatal outcomes of meningococcal disease in the state were concentrated in 1995.

The above description clearly demonstrates that the vaccination was not done at random because exposure - as measured indirectly by disease incidence in the previous year - was used explicitly as a criterion to vaccinate. Although individual effectiveness of the vaccine can be misleading in this situation, population vaccine effectiveness can be calculated as 1 minus the relative risk of a randomly selected person to develop the disease when the population in which vaccination took place is compared to a similar population without vaccination [15]. The key question is what would have happened in the vaccinated population had it not been vaccinated - a hypothetical quantity which may be estimable from the attack rates in the study population or from previous outbreaks [15]. If the population of both vaccinated and unvaccinated areas have been followed-up some time before and after the vaccination, the logic of a cohort study nested within a case-control study can be applied, assuming that the unvaccinated population is a suitable control for the vaccinated one.

However, the vaccinated and unvaccinated populations were very different regarding the risk of developing meningococcal disease - the very reason to vaccinate or not. Thus, a suitable measure of population vaccine effectiveness in this situation should take into account both the temporal aspect (before and after the vaccination) and area-specific characteristics, including differential exposure (vaccinated versus unvaccinated population). It is more realistic to think of the unvaccinated population in this context as a comparison rather than a control group, as defined in case-control studies. An effectiveness measure of this type can be interpreted as relative effectiveness compared to the reference group. Instead of using an ideal setting such as RCT as a reference, a specific health care setting within a defined area or health authority can be chosen for this purpose. The relative risks before and after vaccination for each setting can be calculated, and then compared. For example, population effectiveness of vaccination programs can 
be compared between countries using one of them as reference, providing that the case definition is reasonably accurate and unbiased with respect to the countries compared. If these conditions are difficult to satisfy in a daily routine of some health settings, additional resources should be made available for such studies.

Two recent review articles covered a range of important methodological issues but emphasized vaccine efficacy, rather than effectiveness [16,17]. This work aims at developing a measure of vaccine effectiveness suitable for the comparison of changes in disease incidence in unvaccinated versus vaccinated populations when differential exposure is explicitly used as a criterion to vaccinate or not.

\section{Materials and Methods}

All laboratory confirmed cases of meningococcal disease caused by serogroup $\mathrm{C}$ in the state of Santa Catarina one year before and one year after the vaccination against this serogroup were included in the analysis. The cases were selected from the state health authorities' database of all suspect cases based on clinical examination (39.8\%), latex agglutination (9.4\%), bacteriologic examination using Gram's stain (19.6\%), and bacterial culture (31.2\%). The database is part of the national surveillance system of infectious diseases. Federal legislation obliges health professionals in Brazil to report any suspected case of meningococcal disease. The vaccination took place in the last two weeks of March, 1996, so the period from April 1, 1995, to March 31, 1996, was compared to the period from April 1, 1996, to March 31, 1997. The polysaccharide vaccine applied against serogroup $\mathrm{C}$ meningococci was produced by the Bio-Manguinhos laboratory, Fundação Oswaldo Cruz, Rio de Janeiro, under technical supervision of Institute Pasteur Merrieux, Lyon, France. A single dose containing $50 \mu \mathrm{g}$ of the organism was applied subcutaneously. The vaccine coverage was reported to be $100 \%$, reaching 976,389 children and adolescents between 6 months and 14 years of age in the municipalities selected for vaccination. Thirty of the municipalities were in a densely populated coastal area of the state, five in the highland area, and seven further west near the border with Argentina and Uruguay.

The ratio of the cumulative incidence of meningococcal disease among the unvaccinated and vaccinated populations was calculated separately for the period of 1 year before (denoted $R_{1}$ ) and one year after the vaccination campaign $\left(\right.$ denoted $\left.R R_{2}\right)$. The $95 \%$ confidence interval of the ratio was calculated using exact binomial limits for the ratio of 2 Poisson counts [18].

We define the ratio of the rate ratios (RRR) before and after the vaccination campaign as $R R_{1} / R R_{2}$. This ratio can be seen as a ratio of two random variables whose variance can be approximated by the formula showed in Figure 1 [19].

The formula in Figure 1 is a reasonable approximation when the denominator of the ratio has a small coefficient of variation [19]. The variance estimate was obtained by taking the values of $R R_{1}$ and $R R_{2}$ for the expectations of $R R_{1}$ and $R R_{2}$ respectively, and by taking the sample variances of rate ratios as unbiased population estimates.

The efficacy of the vaccination campaign was estimated by (1 - RRR)*100, which is equivalent to (1 $\left.-R_{1} / R_{2}\right) * 100$. This interpretation was based on the definition of $R R_{1}$ and $R R_{2}$ as measures of relative risk in unvaccinated relative to vaccinated area, so their ratio $\mathrm{RR}_{1} / \mathrm{RR}_{2}$ can be seen as a measure of change in risk ratio after vaccination compared to before vaccination. Assuming that vaccine is effective in a targeted area vaccination campaign and that disease incidence in the unvaccinated area is close to zero, the incidence rate ratio between unvaccinated and vaccinated areas should become closer to the value of one and the ratio of this measure before and after vaccination shows by how much the incidence rate ratio has changed, presumably due to vaccination. Both direct and indirect vaccination effects in both areas are taken into account with this approach.

The choice of denominator for the rate ratio is arbitrary from the interpretation point of view. Here, the ratio of unvaccinated to vaccinated areas was convenient to underline by how much the disease 
Figure 1.

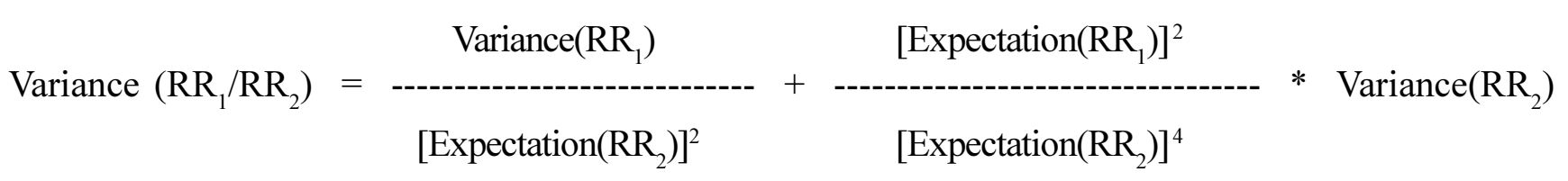

incidence was lower in the former compared to the latter, thus showing the rationale for a targeted area vaccination campaign.

\section{Results}

Relevant epidemiological data for the vaccine effectiveness calculation are presented in Table 1 . The $95 \%$ confidence intervals do not overlap for the age group between 6 months and 14 years and for the general population, thus indicating a statistically significant effect of the vaccination for these categories. This justifies the calculation of the $R R R=R R_{1} / R_{2}$ and its $95 \%$ confidence intervals. In the general population, $\mathrm{RR}_{1} / \mathrm{RR}_{2}=0.257(0.004-0.473)$ and vaccine effectiveness, defined as $\left(1-\mathrm{RR}_{1} / \mathrm{RR}_{2}\right) * 100$, is $74.3 \%$ (52.7-99.6\%). For the children between the ages 6 months and 14 years, $R_{1} / R_{2}$ is $0.069(0.000-0.148)$ and vaccine effectiveness is $93.1 \%$ (85.2-100\%).

\section{Discussion}

The interpretation of the RRR is pretty straightforward in statistical terms. It extends the logic of a rate ratio to a two-step calculation where the first step is the rate ratio between unvaccinated and vaccinated populations and the second step is before versus after ratio of the first step result. The numerator and denominator can be switched both in the first and in the second step without loss of generality. For example, it may be convenient to calculate rate ratio of disease incidence in vaccinated compared to unvaccinated population and divide this quantity for the period after the vaccination with the corresponding value for the period before the vaccination. Similarly, it may be more intuitive to compare before and after rate ratio for vaccinated and unvaccinated populations. In Santa Catarina, the incidence of meningococcal disease per 100,000 inhabitants dropped from 3.15 to 1.09 in the vaccinated area, while it increased from 0.73 to 0.99 in the unvaccinated area (Table 1). If we take the ratio of the after versus before incidence rate ratio for each area, the comparison is between 0.346 and 1.356 , the ratio of which equals 0.255 . One minus the latter indicates $74.5 \%$ population vaccine effectiveness, which is the same as obtained in the results section for the general population (within the rounding error range). However, more stable quantities should be taken for the denominator to reduce the variance of the rate ratios and RRR.

The utility of RRR in a decision making process may be limited by the choice of the reference group and by its summary nature. The more specific the former, the more difficult it is to generalize the findings to other settings. The summary nature of this effectiveness measure is based on taking into account both time (before and after) and area-specific change (unvaccinated and vaccinated), as well as by not distinguishing between direct and indirect effects. When unvaccinated and vaccinated populations mix, RRR will underestimate indirect vaccine effects, particularly in high incidence areas. This is due to the multiplicative nature of the carry-over effect in high incidence areas because of the increased prevalence of meningococci carriers and, therefore, higher probability of exposure. To illustrate this point, we cite Davies, et al. [20] who 


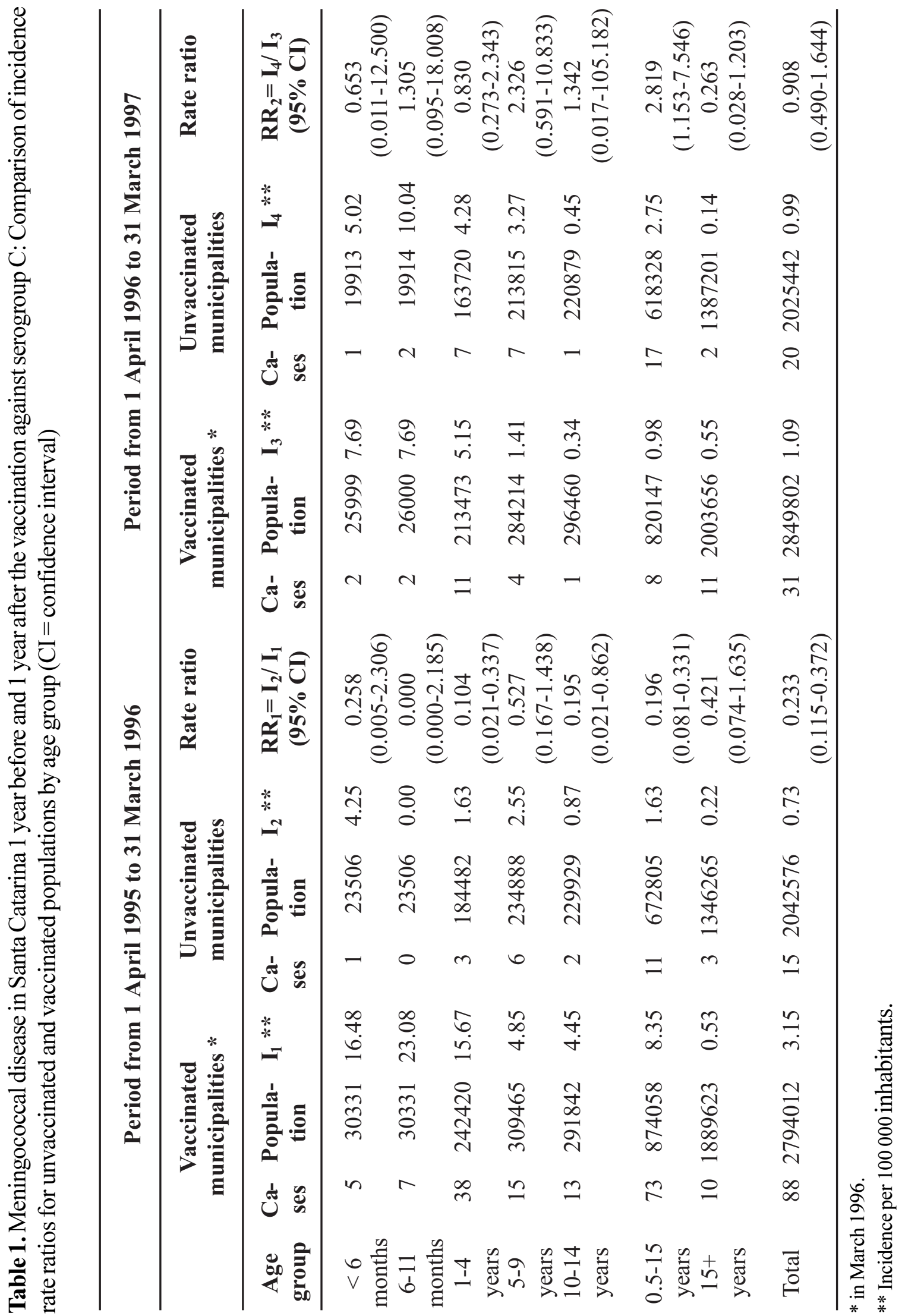


pointed out that the percentage of meningococci carriers in the population was estimated in the range of $5 \%$ to $10 \%$ when the disease incidence was between 1 and 2 cases per 100,000 inhabitants but reached half of the population during a community outbreak.

During the period analyzed, approximately $40 \%$ of the notified cases of meningococcal disease were serogrouped. The case-fatality among the latter was between three and four times that of the cases not serogrouped, thus indicating increased probability of serogrouping less grave cases as pointed out by Costa $[10,13]$. However, the decision to vaccinate was not related to this selection mechanism. We could not quantify the surveillance system reporting bias where grave cases could have been reported more likely. It was thought that the gravity of meningococcal disease and thus the need for medical treatment considerably reduced this bias.

A small number of cases increased the variability of RRR, particularly within the specified age groups. Longer follow-up may help in this situation but too long a period may lose sight of important changes such as serogroup B expanding at the expense of serogroup $\mathrm{C}$ after the vaccination. Among the cases serogrouped, the former increased from $43.8 \%$ in 1995 , to $78.9 \%$ in 1998 , while the latter reduced its presence from $54.7 \%$ in 1995 , to $20 \%$ in 1998 .

Despite above limitations, overall bias in serogroup $\mathrm{C}$ case detection was not believed to be large compared to the beneficial effects of the vaccination campaign captured by RRR. The measures of population effectiveness of vaccination were shown to be more robust with respect to non-uniform vaccination, changes in contact patterns by vaccinees, and ability of the vaccine to reduce infectiousness compared to the measures of individual vaccine effectiveness [15]. In addition, the choice of a 1-year period before and after the vaccination avoids the effect of seasonal variations on meningococcal disease incidence. Considering all these factors, we conclude that the antimeningococcal vaccine against serogroup $\mathrm{C}$ in the state of Santa Catarina was effective for the general population and particularly for those under 15 years of age, although it could not be confirmed in more narrowly defined age bands, probably due to the lack of statistical power.

The lack of immunogenicity of anti-meningococcal polysaccharide vaccines in early infancy-exactly when children are more susceptible to the disease - seems to have been resolved by recently developed conjugate vaccines $[21,22]$. The use of polysaccharide vaccines against both $\mathrm{B}$ and $\mathrm{C}$ serogoups meningococci for children under 4 years of age was shown to be efficient in reducing the incidence of meningococcal disease in the state of Santa Catarina [10], but not in Rio de Janeiro [9] and São Paulo [11] in the early 1990s. However, not even the Santa Catarina study [10] showed a statistically significant reduction in meningococcal disease incidence due to either serogroup B or C separately, but only that due to the combined effect of the two in this age group. Outside Brazil, a Canadian study [5] confirmed effectiveness of antimeningococcal polysaccharide vaccine against serogroup $\mathrm{C}$ for individuals under 20 years of age, underlying the importance of indirect vaccine protection. This interpretation was corroborated with a Spanish experience with polysaccharide vaccine against serogroup $\mathrm{C}$, where bactericide activity for vaccinated children under 4 years of age was found to be very low, in contrast with very good effectiveness results [8]. A systematic review of the polysaccharide vaccines against serogroup $\mathrm{C}$ meningococci concluded that the vaccine was not effective under 2 years of age and, therefore, not suitable for routine immunization, but was indicated for controlling outbreaks [7].

Vaccination strategies should take into account relevant epidemiological, health service, social, and cultural characteristics of a particular setting, as demonstrated through examples from the African continent with a predominance of A serogroup meningococci [23-26]. Monitoring the changes in serogroup profile is essential to design a cost-effective strategy. To this end, suspected cases can be routinely tested by relatively inexpensive procedures such as counterelectroimmunophoresis and sporadically verified by more specific tests such as polymerase chain reaction [27]. The latter may provide more precise 
estimates of infectious agent dynamics, thus enabling better targeting of available resources. Under these circumstances, a simple measure of effectiveness such as RRR may greatly contribute to decisions on vaccination strategies, even when the data are collected in less controlled environments such as daily routine of health services in a particular setting.

\section{References}

1. Amato Neto V., Baldy J.L.S., Silva L.J. Imunizações efetuadas circunstacialmente no Brasil. São Paulo: Sarvier, 1991.

2. Ministério de Saúde. Guia de Vigilância Epidemiológica. Brasília: Fundação Nacional de Saúde, Centro Nacional de Epidemiologia, 1994.

3. Ministério de Saúde. Programa Nacional de Meningites. Relatório do Informe epidemiológico do SUS. Brasília: Fundação Nacional de Saúde, Centro Nacional de Epidemiologia, 1999.

4. Rico C.O., Pereira C.C., Fernandez A.A. Eficacia poslicenciamiento de VA-MENGOC-BC en menores de 6 anos en Holguin, Cuba. Primer ano de observacion. Rev Cubana Med Trop 1995;47(1):59-64.

5. De Wals P., Dionne M., Douville-Fradet M., et al. Impact of a mass immunization campaign against serogroup $\mathrm{C}$ meningococcus in the Province of Quebec, Canada. Bull World Health Organ 1996;74(4):407-11.

6. Sifontes S., Infante J.F., Perez P., et al. The hyperferremic mouse model for the evaluation of the effectiveness of VA-MENGOC-BC against Neisseria meningitidis B clinical isolates. Arch Med Res 1997;28(1):41-5.

7. Gonzalez E.J., Garcia C.L., Alcaide J.J.F., et al. Eficacia de la vacuna meningococica de polisacarido capsular del grupo C. Rev Esp Salud Publica 1997;71(2):103-26.

8. Gonzalez A.L., Garcia M.J. Seroprevalencia de anticuerpos bactericidas frente a meningococo $\mathrm{C}$ em Cantabria 10 meses despues de la campana de vacunacion. Rev Esp Salud Publica 1998;72(4):365-74.

9. Noronha C.P., Struchiner C.J., Halloran M.E. Assessment of the direct effectiveness of $\mathrm{BC}$ meningococcal vaccine in Rio de Janeiro, Brazil: a case-control study. Int J Epidemiol 1995;24(5):1050-7.

10. Costa E.A., Martins H., Klein C.H. Avaliação da proteção conferida pela vacina antimeningocócica BC no Estado de Santa Catarina, Brasil, 1990/92. Rev Saúde Pública 1996;30(5):460-70.

11. Moraes J.C., Perkins B.A., Camargo M.C.C., et al. Protective efficacy of serogroup B meningococcal vaccine in São Paulo, Brazil. Lancet 1992;2:1074-8.
12. Clemens J., Brenner R., Rao M., et al. Evaluating new vaccines for developing countries: efficacy or effectiveness. JAMA 1996;275(5):390-7.

13. Costa E.A. Effectiveness of meningococcal vaccine in Brazil (letter). Int J Epidemiol 1997;26(3):681-4.

14. Secretaria de Estado da Saúde de Santa Catarina. Estatísticas de Saúde. [cited 25 March 2001] Available from URL:http://www.saude.sc.gov.br

15. Haber M., Watelet L., Halloran M.E. On individual and population effectiveness of vaccination. Int $\mathrm{J}$ Epidemiol 1995;24(6):1249-60.

16. Rodrigues L.C., Smith P.G. Use of the case-control approach in vaccine evaluation: efficacy and adverse effects. Epidemiol Rev 1999;21(1):56-72.

17. Halloran M.E., Longini I.M.Jr., Struchiner C.J. Design and interpretation of vaccine field studies. Epidemiol Rev 1999;21(1):73-88.

18. Daly L.E., Bourke G.J., McGilvray J. Interpretation and uses of medical statistics. Oxford, UK: Blackwell Science, 1996.

19. Armitage P., Berry G. Statistical methods in medical research. Oxford: Blackwell Scientific Publications, 1994.

20. Davies A.L., O'Flanagan D., Salmon R.L., et al. Risk factors for Neisseria meningitidis carriage in a school during a community outbreak of meningococcal infection. Epidemiol Infect 1996; 117:259-66.

21. MacLennan J.M., Shackley F., Heath P.T., et al. Safety, immunogenicity, and induction of immunologic memory by a serogroup $\mathrm{C}$ meningococcal conjugate vaccine in infants: A randomized controlled trial. JAMA 2000;283(21):2795-801.

22. Rennels M.B., Edwards K.M., Keyserling H.L., et al. Safety and immunogenicity of four doses of Neisseria meningitidis group $\mathrm{C}$ vaccine conjugated to CRM197 in United States infants. Pediatr Infect Dis J 2001;20(2):153-9.

23. Varaine F., Caugant D.A., Riou J.Y., et al. Meningitis outbreaks and vaccination strategy. Trans R Soc Trop Med Hyg 1997;91(1):3-7.

24. Miller M.A., Wenger J., Rosenstein N., Perkins B. Evaluation of meningococcal meningitis vaccination strategies for the meningitis belt in Africa. Pediatr Infect Dis 1999;18(12):1051-9.

25. Woods C.W., Armstrong G., Sackey S.O., et al. Emergency vaccination against epidemic meningitis in Ghana: implications for the control of meningococcal disease in West Africa. Lancet 2000;355(9197):30-3.

26. Kaninda A.V., Belanger F., Lewis R., et al. Effectiveness of incidence thresholds for detection and control of meningococcal meningitis epidemics in northern Togo. Int J Epidemiol 2000;29(5):933-40. 
27. Saunders N.B., Shoemaker D.R., Brandt B.L., Zollinger W.D. Confirmation of suspicious cases of meningococcal meningitis by PCR and enzyme-liked immunosorbent assay. J Clin Microbiol 1997;35(12):3215:9. 